\title{
Logical and Pragmatic Meaning in the Interpretation of Connectives: Scalar Implicatures and "Shallow" Processing
}

\author{
István Fekete ${ }^{a}$, Mátyás Gerőcs ${ }^{b}$, Anna Babarczyc ${ }^{c}$ and Balázs Surányi ${ }^{d}$ \\ a, b,c, dHungarian Academy of Sciences, Budapest, Hungary; dPázmány Péter Catholic University, \\ Piliscsaba, Hungary \\ aistvfekete@gmail.com; bgerocs.matyas@nytud.mta.hu, cbabarczy.anna@nytud.mta.hu; \\ dsuranyi@nytud.hu
}

\begin{abstract}
On one prominent view, endorsed by several authors pursuing neo-Gricean approaches, scalar implicatures like "but not both" in the exclusive interpretation of the conjunction or "A or B but not both," are generated automatically by default in the absence of context. By contrast, the contextualist view holds that scalar implicatures arise only when licensed by the context. We addressed this dispute by performing a sentence-picture verification task experiment, comparing the processing of two connectives in Hungarian: és ("and") and vagy ("or"). Crucially, the verification task required only a shallow processing of the meaning of target sentences. The results suggest that in such a task, while the entailment of the connective and was computed automatically, the implicature of or was not activated. This finding speaks against defaultism, and favors contextualist approaches to generalized conversational implicatures.
\end{abstract}

Keywords: connectives; disjunction; implicature; shallow processing; contextualism; Relevance Theory

\section{Defaultist and Contextualist Approaches to Scalar Implicatures}

On one prevailing view, endorsed by some prominent work pursuing a neo-Gricean approach (e.g., Levinson 2000; Landman 2000; Chierchia 2004), scalar implicatures such as "but not both" in the exclusive interpretation of the conjunction or "A or B but not both" are generated automatically by default. One may distinguish between a strong version of defaultism, according to which implicatures are not effortful at all (e.g., Levinson 2000) and a weak version, according to which implicatures arise by default (even when a context is lacking) but they nevertheless (may) incur extra processing cost. On another, equally influential view, scalar implicatures only arise when required by the context (e.g., Sauerland 2004; Van Rooij and Schulz 2004; Geurts 2011). The latter, contextualist view is advocated by Relevance Theory (RT), according to which scalar implicatures are generated only in contexts in which they are relevant in the technical sense that they yield a significant cognitive effect at a reasonable processing cost (Sperber and Wilson 1995; Wilson and Sperber 2012; Carston 1998). Although some recent psycholinguistic experiments, performed to assess the opposing predictions of these two major theories of the way scalar implicatures arise, apparently disfavor the defaultist view (Noveck and Posada 2003; Bott and Noveck 2004; Breheny, Katsos, and Williams 2006; Katsos 2006; Huang and Snedeker 2009; Zondervan 2010), the results have been contested 
(Feeney et al. 2004; Degen et al. 2009; Grodner et al. 2010; see also Zondervan's [2010] methodological criticism of some of the experiments that have been interpreted as supporting the contextualist view).

\section{A Previous Experiment on the Processing of Disjunction}

An experimental study of particular relevance to our own is Chevallier et al. (2008). ${ }^{1}$ This investigation assessed in three experiments the effects of contrastive stress on the disjunction or. Disjunctions can be enriched from a logical reading (the inclusive meaning) to an exclusive reading (A or B "but not both"). Chevallier and her colleagues demonstrated that both a visual and prosodic contrastive focus on or affected the enrichment.

It is a common view, also shared by Relevance Theory, that implicatures in general (including the exclusive implicature at hand) arise in an effortful manner. Assuming that visual or prosodic prominence makes the disjunctive expression (more) relevant in a Relevance Theoretic sense, by making the processing of the disjunction less costly (see also Van Rooij and Schulz [2004] on how focus facilitates scalar implicatures), Chevallier and her colleagues predicted that sentences such as You can have the meat course or the fish course will be interpreted with a lower rate of exclusive implicatures than You can have the meat course OR the fish course, with the disjunction marked as prominent visually or prosodically. Indeed, they found that making or prominent significantly increased the proportion of exclusive readings (cf. also Zondervan 2010). This result speaks in favor of contextualist approaches to scalar implicatures, as it shows that their generation is affected by prominence relations in the sentence. ${ }^{2}$

The strategy of Chevallier at al.'s experiment was to try to facilitate implicatures. Empirical studies in the realm of the psychopragmatics of implicatures often seek to do the opposite, i.e., to reduce the likelihood of implicatures through an experimentally increased processing load. In the experiment to be presented in this paper, we employed a strategy different from both of these: our experiment examined the effect of decreasing cognitive effort on the computation of a scalar implicature.

\section{The Experiment}

\subsection{The Paradigm}

The experimental task involved a form of "shallow" processing (cf. the concepts of "goodenough," or "shallow" cognitive representations in experimental work by Ferreira, Bailey, and Ferraro [2002] and Louwerse and Jeuniaux [2010], respectively). We tested the processing

1 See also Paris (1973), whose study contains results from the testing of the (offline) interpretation of disjunction in adult controls. The disjunctions tested by Paris differ from those in our experiment in that they linked two complete propositions rather than two phrases. Pijnacker et al. (2009) report that in a neutral context, normal controls derive the exclusive implicature of disjunction at a rate of $54 \%$. The interpretation of the latter result requires caution, as enforcing the lack of a discourse context in an experimental stimulus is notoriously difficult. When no context is given, subjects may still "project" contexts of their own in the course of processing sentence interpretation.

2 Response times for the exclusive readings were not different, regardless of whether contrastive stress was used or not. In other words, while intonation altered how the sentence was interpreted, it did not alter the time-course for the inference. 
of the connectives és ("and") and vagy ("or") in Hungarian in a sentence-picture verification task similar to those used in the mental simulation literature (e.g., Stanfield and Zwaan 2001; Zwaan, Stanfield, and Yaxley 2002). Each picture was preceded by a sentence describing a scenario with two objects appearing as NPs conjoined either by and or by or (Connective Type), e.g., John peeled the orange and/or the banana. The state of the two objects either matched or mismatched (Congruence) the scenario explicitly described in the previous sentence. For example, in the mismatching condition of and-sentences only one of the two objects was peeled (incongruently with the entailment of and). In the case of $o r$-sentences, both objects were peeled in the mismatching condition (incongruently with the implicature of or). Figure 1 illustrates the crucial manipulation of the objects in our experiment. The upper set of pictures is consistent with the implicature of $o r$, viz. "and not both," while the one below it is consistent with the logical meaning of or ("and maybe both").
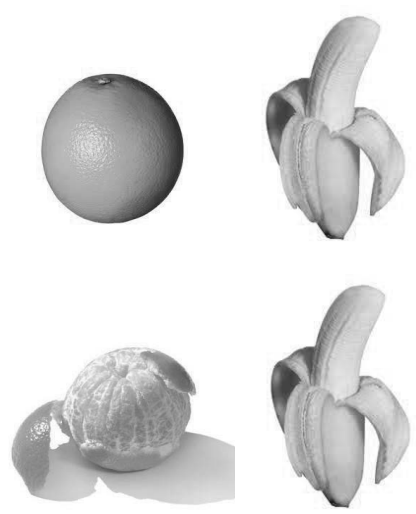

Figure 1. Example of congruent versus incongruent picture stimuli in our experiments. Test sentence:

John peeled the orange or the banana.

The participants' task was unrelated to both Connective Type and Congruence: they had to decide if both of the two objects had been mentioned in the previous sentence or not (without considering the states of the objects depicted). The dependent measure was response time to picture stimuli.

As the implicature associated with or would not yield any cognitive effect in terms of the task itself, on Relevance Theoretic assumptions it is predicted not to arise. By contrast, on the defaultist view (whether the strong or the weak version is considered) the implicature is expected to be generated automatically.

The experimental paradigm that we adopted deserves a comment before we describe the experiment in more detail. As already pointed out, the task we employed requires only "shallow" processing of the test sentences. Our design is probably the closest to that used by Stanfield and Zwaan (2001), who asked participants to decide whether or not pictures depicted the actions described in previously presented sentences. The actions described either a vertical or horizontal orientation, such as driving a nail into the wall or into the ceiling. The results showed that their subjects responded more quickly to the pictures that described the same orientation as the action described. Stanfield and Zwaan (2001) conclude that the participants activated perceptual imagery of the action described in the sentence and this caused the effect. 
Along similar lines, Richardson et al. (2003), for example, showed a direct connection between perceptual and conceptual representations. They demonstrated that the comprehension of verbs that encode horizontal or vertical schemas, such as push, evokes spatial representations. The processing of such verbs interacted with shape discrimination along the horizontal or vertical axis. Other investigations also demonstrate that motion words affect the detection and perception of visual motion (e.g., Kaschak et al. 2005). Scorolli and Borghi (2007) asked their subjects to judge whether sentences containing a verb and a noun made sense. Participants had to respond either by pressing a pedal or speaking into a microphone. The verbs described actions that were performed with the mouth, hands, or the feet. Results showed that response times with the microphone were fastest with sentences encoding "mouth-verbs" and response times with the pedal were fastest with sentences encoding "foot-verbs."

The general interpretation of this body of experimental evidence is that words evoke analogous perceptual and motor representations that are associated with the real-world referents of the words that they refer to, and this affects reaction times. What is significant from the perspective of our own experiment is that even in such "shallow" processing tasks, rather subtle differences in meaning actually get processed, as revealed by the response time data.

\subsection{Method}

\subsubsection{Participants}

Seventy-seven Hungarian students from Budapest participated in the experiment for course credit (Mean age: 22.5, Age range: 17-32; 33 female and 44 male participants). The data of seven participants were discarded because their overall accuracy was under $75 \%$. All the participants were native speakers of Hungarian.

\subsubsection{Stimuli}

32 critical sentences and 64 filler sentences were constructed. Critical trials (a sentence containing a connective and a picture stimulus with the objects) required an affirmative response to a yes/no question, while filler sentences required a negative response in 48 trials, and in 16 trials an affirmative response. In other words, in half of the trials the picture stimuli required a positive response, and in the other half a negative response. None of the sentences was ambiguous in terms of meaning.

The critical sentences were counterbalanced in four between-subject lists. These four pseudo-randomly organized intra-group lists were created in order to counterbalance items and conditions (incomplete counterbalancing technique). Each list included one of four possible versions, with 20 participants assigned to each list randomly. Each participant saw only one list, and each participant read each sentence once. Each sentence was presented with every Connective Type (and/or) across the experiment. Each item was tested equally often in each condition, and each subject received an equal number of items in each condition. Because lists were included only to reduce error variance, effects involving lists are not discussed in further detail. Reading time data were collapsed across lists. 


\subsubsection{Procedure}

Participants were first presented with an instruction screen. They were asked to read the sentences that appeared on the computer screen and press the SPACE key if they had read the sentence. They were told that after every sentence they would see a picture with two objects; their task was to decide if both of the objects had been mentioned in the previous sentence or not. They were also instructed not to pay attention to the state of the objects. Each participant was tested individually in one session lasting approximately 12 minutes. Participants first completed a practice phase, in which they were familiarized with the logic of the experiment. One trial consisted of a sentence and a picture stimulus. The sentence appeared in the center of the center of the computer screen. After the participant read the sentence, a picture appeared. The mean response times to the picture stimuli were collected. The sentences appeared one after the other with a fixation cross appearing between trials for $1000 \mathrm{~ms}$. There was no set limit on response time at the point of the pictures or the sentences; however, subjects were asked to react to picture stimuli as quickly as possible because verification time was measured at the point when the pictures were shown. The trials were randomized across participants. We used E-Prime to run our experiment.

\section{Results and Discussion}

The practice trials were excluded from the analyses, as were the filler items. Erroneous trials where a wrong answer was given to the picture stimuli - were also excluded from the analyses. The data of seven participants were discarded (overall accuracy under 75\%). Missing values were not replaced cellwise. The means of the median reading times of the critical trials were taken. The picture verification times were the primary focus of the analysis but we were also interested in the reading times of sentence stimuli. The table below illustrates the mean picture verification times (and $S D$ s) in the four conditions:

\begin{tabular}{l|c|c|c}
\hline Condition & Mean (ms) & Standard deviation (SD) & Number of Participants \\
\hline AND_match & 1124.41 & 295.13 & 66 \\
\hline AND_mismatch & 1269.83 & 445.35 & 66 \\
\hline OR_match & 1193.82 & 346.94 & 66 \\
\hline OR_mismatch & 1170.45 & 346.33 & 66 \\
\hline
\end{tabular}

Table 1. Mean verification times (ms) of the picture stimuli in the four conditions.

Mean picture verification times were first analyzed in a participant-based $2 * 2$ ANOVA model with Connective Type (two levels: and/or) and Picture Congruence (two levels: match/mismatch) as within-participants factors. We found a significant interaction between Connective Type and Picture Congruence, $F(1,65)=12.224, p<0.001, \eta_{\mathrm{p}}{ }^{2}=0.158$, indicating that the two connectives behave differently in our experiment. The main effect of Congruence is significant, $F(1,65)=$ $6.825, p=0.011, \eta_{\mathrm{p}}{ }^{2}=0.095$. However, Connective Type does not reveal a significant main effect; $F(1,65)=0.358, p=0.552, \eta_{\mathrm{p}}{ }^{2}=0.005$. Since we found a significant interaction, the criticism that participants just scanned the nouns in the sentences, skimming over the connectives, can be ruled out. The figure below depicts the mean picture verification times in the Connective Type and Picture Congruence conditions: 
Mean Verification Times of Picture Stimuli in the two Connective Type Conditions as a Function of Picture Congruence (matching versus mismatching)

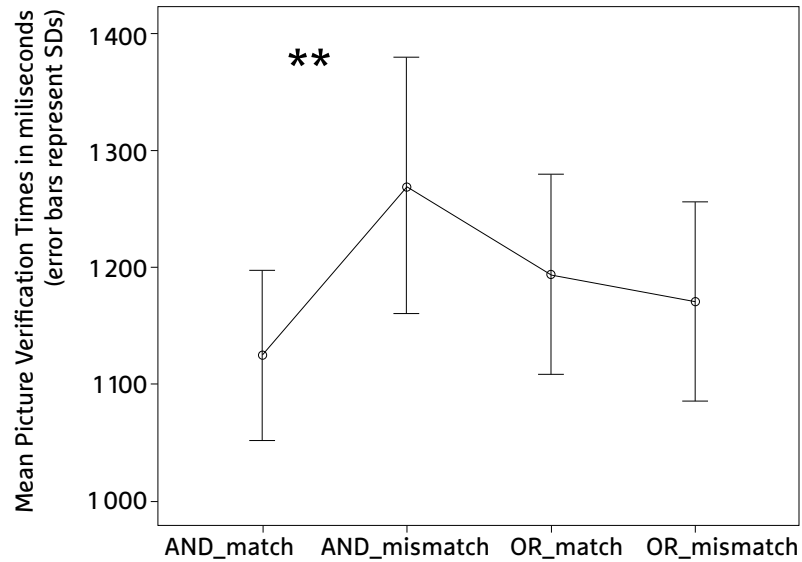

Figure 2. Mean verification times (ms) of the picture stimuli in the two connective type conditions.

Simple effects were also examined, applying Bonferroni-correction: the alpha level of every comparison was adjusted according to the Bonferroni formula, which in our case is tantamount to multiplying the alpha level by four because we were interested in only four critical comparisons. Most importantly, pictures after and-sentences in the matching picture condition were verified significantly faster than mismatching pictures, $t(65)=-3.628, p=0.004$ (Bonferroni corrected). Crucially, however, the same effect was not revealed in the set of or-sentences, $t(67)=0.897, p=0.999$ (Bonferroni corrected; uncorrected $p=0.373$ ).

Mismatches are generally expected to slow down reaction times, compared to the matching condition. This is indeed what happened after and-sentences, but this was not the case after orsentences. The lack of a slow-down after $o r$-sentences is expected if the exclusive implicature of or was not generated. The lack of the exclusive implicature is predicted on the contextualist view, since it was irrelevant to the task. On (both strong and weak) defaultist approaches, the implicature is expected to be generated even in such cases. Then either the mismatch with the picture, or both that and the generation of the implicature itself (see, e.g., Noveck and Posada 2003; Bott and Noveck 2004; Breheny, Katsos, and Williams 2006; De Neys and Schaeken 2007; Huang and Snedeker 2009) would be predicted to cause a slow-down in the or-mismatch condition (cf. Shetreet et al.'s forthcoming fMRI study), contrary to fact.

Pictures in the and-match condition were verified faster than those in the or-match condition, $t(67)=-2.531, p=0.028$ (Bonferroni corrected). Concentrating on just this difference, one could potentially attribute this to the extra processing cost incurred by generating the exclusive implicature of or. However, this interpretation is hard to maintain in view of the fact that was just discussed, namely that the mismatch of the allegedly generated implicature with the picture had no effect. (Furthermore, this interpretation is not even available on a strong defaultist approach, according to which implicature generation is not only automatic, but also has no cost; e.g., Levinson 2000.) Instead, the longer verification times for the or-match condition can be attributed to an essential difference in complexity between the meanings of the conjunction and and the disjunction or. In order to capture the interpretation of disjunctions in modal environments, 
several recent studies have analyzed disjunction as having a more complex meaning than conjunction. According to Zimmermann (2000), the semantics of disjunction can be described as the conjunction of (possibility) modal propositions. Thus, informally speaking, a disjunctive sentence such as "A or B" is interpreted as "Possibly A and possibly B." Others, including Simons (2005) and Alonso-Ovalle (2006), have argued that disjunctions introduce sets of propositional alternatives into the semantic derivation. On either of these two types of approaches, the more complex meaning of the disjunction may arguably take longer to construct and, especially, to verify than that of an ordinary conjunction. (Although no verification related to the interpretation of the conjunction and the disjunction was part of our "shallow processing" task, it is clear from the results discussed thus far that such verification did involuntarily take place during the processing of the sentence-picture stimulus pairs.)

Pictures in the and-mismatch condition were reacted to significantly more slowly than those in the $o r$-mismatch condition, $t(65)=2.638, p=0.02$ (Bonferroni corrected). This difference is explained straightforwardly if there was an actual mismatch between the picture stimulus and the interpretation assigned by the participants to the sentence only in the and-mismatch condition, but not in the or-mismatch condition. There was no genuine discrepancy between the sentence and the picture stimulus in the latter condition if, as we are assuming, the exclusive implicature of the disjunction did not get generated in this task. On defaultist approaches, according to which the exclusive implicature must have been generated in the $o r$-sentences, the longer response time associated with the and-mismatch conditions than with the or-mismatch conditions is difficult to account for. Perhaps one possibility is to assume that in some sense the and-mismatch conditions, in which the mismatch is due to a semantic entailment of the conjunction, lead to a stronger discrepancy than does the implicature of the disjunction. But that assumption rests on dubious grounds in that both the entailments of conjunction and the exclusivity implicature of disjunction are arguably part of the truth conditions of the respective sentence types (see Carston [2004] for a forceful defense of this position). The differences between the two may only lie in how effortful they are to generate or to cancel, but both of these considerations would predict the opposite of what we found, i.e., it is the or-mismatch condition that should have incurred longer response times than the and-mismatch condition.

We also analyzed the mean reading times of the sentences before the picture stimuli to determine if the two Connective Types are processed differently during reading. As or-constructions may in general be associated with an implicature, they could in principle be harder to process in reading than and-constructions. If, contrary to our own assumptions, an exclusivity implicature had been calculated during the reading of the $o r$-sentences, then we might find longer reading times for the $o r$-sentences than for the and-sentences (note that in Hungarian the disjunction itself, i.e., vagy, is even a longer word than the conjunction és). Nevertheless, we found that the and-sentences (Mean: $1980.29 \mathrm{~ms}, S D: 608.22 \mathrm{~ms}$ ) were not read differently from the or-sentences (Mean: $2046.22 \mathrm{~ms}, S D: 601.77 \mathrm{~ms}$ ), $t(67)=-1.324, p=0.190)$. This finding is predicted by our assumption that the disjunction was not associated with an implicature in the experimental task. Note that this result does not argue specifically in favor of that assumption, but it is straightforwardly compatible with it. If one were to assume that the implicature had actually been generated, then the same result could be explained away by assuming that the extra processing cost this incurred did not manifest itself during the reading of the target sentences themselves, which contained the conjoined and disjoined phrases in a sentence-final 
position. Alternatively, a strong defaultist approach, according to which implicatures incur no extra cost, also predicts a lack of difference in reading times.

We close our discussion by mentioning a potential criticism of the experimental task itself. One might suggest that the matching and mismatching picture stimuli differed inherently in terms of verification times, causing some of our results to be confounded. Specifically, it is in principle possible that an unpeeled banana is easier to verify than a peeled one on the basis of the principles of familiarity and prototypicality. This possibility, however, can be excluded. First, a difference in familiarity/prototypicality was present in only some of the pictures. Second, there was no significant difference between matching and mismatching conditions in the or-condition. This is unexpected if the non-prototypicality of mismatching pictures had an effect.

\section{Conclusions}

In this study we reported on the results of an experiment examining the scalar implicature associated with disjunction with the aid of a task requiring "shallow processing." Overall, the results suggest that in such a task, while the entailment of the connective and was computed automatically, the implicature of or was not activated. This finding speaks against (both strong and weak versions of) defaultism, according to which generalized conventional implicatures are generated by default, in the absence of context, and favors contextualist approaches. The results are predicted straightforwardly by basic principles of Relevance Theory, according to which a scalar implicature is more likely to be generated if its relevance, i.e., the ratio of the positive cognitive effect that it can achieve and the processing cost it requires, is high. As the potential positive cognitive effect in our experimental task was extremely low, the implicature was simply not worth processing.

From a broader perspective, our results indicate that "shallow" processing tasks are a promising experimental tool for psycholinguistic experimental research at the semantics/pragmatics interface more generally.

\section{Funding Acknowledgement}

The authors of this work were supported by the Momentum grant of the Hungarian Academy of Sciences, and by grant no. 84217 of the Hungarian Scientific Research Fund.

\section{Works Cited}

Alonso-Ovalle, Luis. 2006. "Disjunction in Alternative Semantics." PhD diss., University of Massachusetts.

Bott, Lewis, and Ira A. Noveck. 2004. "Some Utterances are Underinformative: The Onset and Time Course of Scalar Inferences.” Journal of Memory and Language 51: 437-57.

Breheny, Richard, Napoleon Katsos, and John Williams. 2006. "Are Generalised Scalar Implicatures Generated by Default? An On-Line Investigation into the Role of Context in Generating Pragmatic Inferences. Cognition 100 (3): 434-63.

Carston, Robyn. 1998. "Informativeness, Relevance and Scalar Implicature.” In Relevance Theory: Applications and Implications, edited by Robyn Carston and Seiji Uchida, 179-236. Amsterdam: John Benjamins.

Carston, Robyn. 2004. "Truth-Conditional Content and Conversational Implicature." In The Semantics/Pragmatics Distinction, edited by Claudia Bianchi, 18-48. Stanford: CSLI Publications. 
Chevallier, Coralie, Ira A. Noveck, Tatjana Nazir, Lewis Bott, Valentina Lanzetti, and Dan Sperber. 2008. "Making Disjunctions Exclusive." Quarterly Journal of Experimental Psychology 61 (11): 1741-60.

Chierchia, Gennaro. 2004. "Scalar Implicatures, Polarity Phenomena and the Syntax/Pragmatics Interface.” In Structures and Beyond: The Cartography of Syntactic Structures, Vol. 3, edited by Adriana Belletti, 39-103. Oxford: Oxford University Press.

Degen, Judith, Patricia A. Reeder, Kathleen Carbary, and Michael K. Tanenhaus. 2009. "Using a Novel Experimental Paradigm to Investigate the Processing of Scalar Implicatures." Paper presented at the Experimental Pragmatics Conference 2009, Lyon, April 23-25.

De Neys, Wim, and Walter Schaeken. 2007. "When People Are More Logical under Cognitive Load: Dual Task Impact on Scalar Implicature.” Experimental Psychology 54 (2): 128-33.

Feeney, Aidan, Susan Scafton, Amber Duckworth, and Simon J. Handley. 2004. "The Story of Some: Everyday Pragmatic Inferences by Children and Adults." Canadian Journal of Experimental Psychology 58 (2): 121-32.

Ferreira, Fernanda, Karl G. D. Bailey, and Vittoria Ferraro. 2002. "Good-Enough Representations in Language Comprehension." Current Directions in Psychological Science 11 (1): 11-15.

Geurts, Bart. 2011. Quantity Implicatures. Cambridge: Cambridge University Press.

Grodner, Daniel J., Natalie M. Klein, Kathleen M. Carbary, and Michael K. Tanenhaus. 2010.

“'Some,' and Possibly All, Scalar Inferences Are Not Delayed: Evidence for Immediate Pragmatic Enrichment.” Cognition 116 (1): 42-55.

Huang, Yi Ting, and Jesse Snedeker. (2009). "On-Line Interpretation of Scalar Quantifiers: Insight into the Semantic-Pragmatics Interface.” Cognitive Psychology 58 (3): 376-415.

Kaschak, Michael P., Carol J. Madden, David J. Therriault, Richard H. Yaxley, Mark Aveyard, Adrienne A. Blanchard, and Rolf A. Zwaan. 2005. "Perception of Motion Affects Language Processing." Cognition 94 (3): B79-B89.

Katsos, Napoleon. 2006. "Experimental Investigations of the Effects of Structure and Context on the Generation of Scalar Implicatures." PhD diss., University of Cambridge.

Landman, Fred. 2000. Events and Plurality. Dordrecht: Kluwer.

Levinson, Stephen C. 2000. Presumptive Meaning: The Theory of Generalized Conversational Implicature. Cambridge, MA: MIT Press.

Louwerse, Max M., and Patrick Jeuniaux. 2010. "The Linguistic and Embodied Nature of Conceptual Processing.” Cognition 114 (1): 96-104.

Noveck, Ira, and Andres Posada. 2003. "Characterizing the Time Course of an Implicature: An Evoked Potentials Study." Brain and Language 85 (2): 203-10.

Paris, Scott G. 1973. "Comprehension of Language Connectives and Propositional Logical Relationships." Journal of Experimental Child Psychology 16 (2): 278-91.

Pijnacker, Judith, Peter Hagoort, Jan Buitelaar, Jan-Pieter Teunisse, and Bart Geurts. 2009. "Pragmatic Inferences in High-Functioning Adults with Autism and Asperger Syndrome." Journal of Autism and Developmental Disorders 39: 607-18.

Richardson, Daniel C., Michael J. Spivey, Lawrence W. Barsalou, and Ken McRae. 2003. "Spatial Representations Activated during Real-Time Comprehension of Verbs." Cognitive Science 27 (5): 767-80. 
Sauerland, Uli. 2004. "Scalar Implicatures in Complex Sentences." Linguistics and Philosophy 27: 367-91.

Scorolli, Claudia, and Anna M. Borghi. 2007. "Sentence Comprehension and Action: Effector Specific Modulation of the Motor System.” Brain Research 1130 (1): 119-24.

Shetreet, Einat, Gennaro Chierchia, and Nadine Gaab. Forthcoming. "When 'Some' Is Not 'Every': Dissociating Scalar Implicature Generation and Mismatch." Human Brain Mapping.

Simons, Mandy. 2005. "Dividing Things Up: The Semantics of or and the Modal/or Interaction." Natural Language Semantics 13: 271-316.

Sperber, Dan, and Deirdre Wilson. 1995. Relevance: Communication and Cognition. 2nd ed. Cambridge: Blackwell.

Stanfield, Robert A., and Rolf A. Zwaan. 2001. "The Effect of Implied Orientation Derived from Verbal Context on Picture Recognition.” Psychological Science 12 (2): 153-56.

Van Rooij, Robert, and Katrin Schulz. 2004. "Exhaustive Interpretation of Complex Sentences." Journal of Logic, Language and Information 13: 491-19.

Wilson, Deirde, and Dan Sperber. 2012. Meaning and Relevance. Cambridge: Cambridge University Press.

Zimmermann, Thomas Ede. 2000. "Free Choice Disjunction and Epistemic Possibility." Natural Language Semantics 8:255-290.

Zondervan, Arjen. 2010. Scalar Implicatures or Focus: An Experimental Approach. Utrecht: LOT Publications.

Zwaan, Rolf A., Robert A. Stanfield, and Richard H. Yaxley. 2002. "Language Comprehenders Mentally Represent the Shapes of Objects.” Psychological Science 13 (2): 168-71. 